\title{
A Case of Glomangiopericytoma of the Nasal Septum
}

\section{FM Hanege ${ }^{1}$, A Livaoglu ${ }^{2}$}

\section{Affiliations:}

${ }^{1}$ Department of Otorhinolaryngology, Istanbul Medeniyet University School of Medicine, Goztepe Education and Research Hospital, Istanbul, Turkey

${ }^{2}$ Trabzon Kanuni training and research hospital, Department of pathology, Trabzon

Turkey

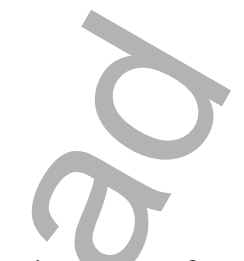

Corresponding Author: Fatih Mehmet Hanege, Assistant Professor

Department of Otorhinolaryngology

Istanbul Medeniyet University School of Medicine,

Goztepe Education and Research Hospital, Kadikoy, 34030, Istanbul, Turkey.

Fax: +90212 2874002

Phone : +90 5325026239

E-mail: hanege@hotmail.com

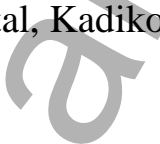

Keywords: glomangiopericytoma, nasal septum, nasal mass, epistaxis. 
The Editor,

Sir,

A 78-year-old female patient presented to our clinic with congestion in the right nasal cavity and recurrent epistaxis for a month period. The medical history was not significant except hypertension, hyperlipidemia and rheumatoid arthritis. On physical examination, there was a $15 \mathrm{~mm}$ diameter, painless and bleeding tumour at the right side of the nasal cavity (Figure 1-a). Endoscopic examination revealed a hemorrhagic mass in the right nasal septum. At labarotory evaluation there was not abnormal results. The hemorrhagic mass of the septum was widely excised endoscopically including the septum mucosa and the perichondrium. In immunohistochemical assessment, Vimentin was (+), SMA (+) and CD31 (+); CD34 focal positive; Desmin (-), S-100 (-), HMB-45 (-) and CK (-) and Ki-67 index was under 1\% (Figure-2a-b-c-d-e). The tumour was histopathologically diagnosed as glomangiopericytoma. During the 15 months of patient follow-up, no signs of recurrence were observed (Figure 1-b).

Glomangiopericytoma, which is a type of sinonasal hemangiopericytoma, arises from the pericytes surrounding capillaries. Glomangiopericytoma is a very rare type of nasal cavity tumour, which shows a very slight rise in the female sex, and incidence age peak is during the seventh decade of life and varies in malignancy potential $(1,2)$. This tumour was classified as glomangiopericytoma by The World Health Organization (WHO) in 2005 (3). Glomangiopericytoma is described in WHO classification as a borderline low malignancy tumour. The local recurrence rate is $16.8 \%$. However recurrence can occur as a result of incomplete excision. Thus recurrence is better considered as residual disease in such cases (4). Differential diagnosis includes similar 
pattern soft tissue tumours such as a solitary fibrous tumour, sinovial sarcoma, hemangioendotelioma, glomus tumour and conventional haemangiopericytomas (5). Malignancy in glomangiopericytomas is uncommon and malignant tumours display nuclear pleomorphism, high mitotic activity, and necrosis (4). In our immunohistochemical assessment, Vimentin was (+), SMA (+) and CD31 (+); CD34 focal positive; Desmin (-), S-100 (-), HMB-45 (-) and CK (-) and Ki-67 index was under $1 \%$. These results diagnosed the tumour as Glomangiopericytoma. The gold standard treatment is usually accepted as resection with tumour-free margins (5). In our case, the tumour was widely excised including the septum mucosa and perichondrium. In our case, during the 15 months of patient follow-up, no signs of recurrence were observed.

Glomangiopericytoma is rarely detected at the nasal septum. Definitive diagnosis should be support with clinical examination and histopathologic and immunohistochemical features. This rarely seen tumour should be kept in mind when evaluating nasal masses. Wide local excision is almost always sufficient for treatment but close follow up is extremely important. 


\section{Referances}

1. Kanazawa T, Nishino H, Miyata M, Kuriki K, Abe K, Ichimura K. Haemangiopericytoma of infratemporal fossa. J Laryngol Otol 2001;115:77-9

2. Thompson LD. Sinonasal tract glomangiopericytoma (hemangiopericytoma). Ear Nose Throat J 2004;83:807.

3. Barnes L, Eveson JW, Reichart P, Sidransky D, Eds. World Health Organization Classification of Tumours. Pathology and Genetics of Head and Neck Tumours. IARC Press: Lyon 2005.

4. Thompson L, Miettinen M, Wenig B. Sinonasal-type hemangiopericytoma: a clinicopathologic and immunophenotypic analysis of 104 cases showing perivascular myoid differentiation. Am J Surg Pathol 2003;27:737-49.

5. Hanege FM, Uzun L, Yavuz C, Ozkanli S, Kurtgoz S. Epithelioid hemangioendothelioma of the nasal septum. B-ENT 2016;12:155-157. 

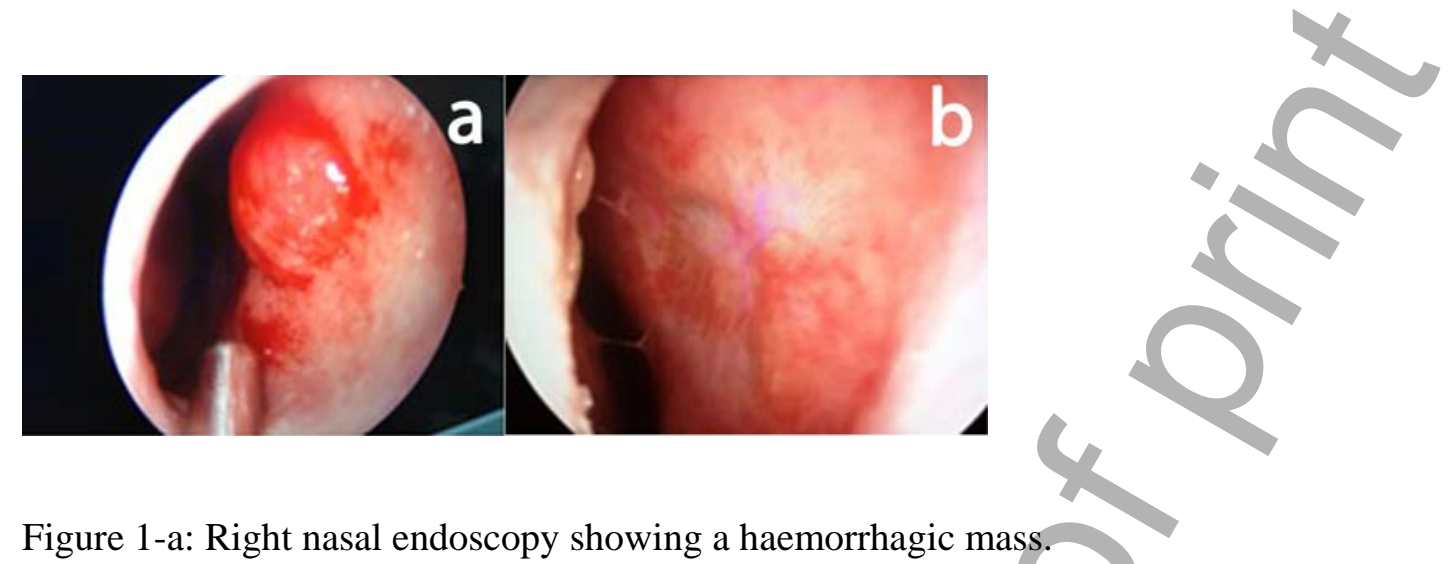

Figure 1-a: Right nasal endoscopy showing a haemorrhagic mass.

Figure1-b: The 15 months of patient follow-up, no signs of recurrence were observed.

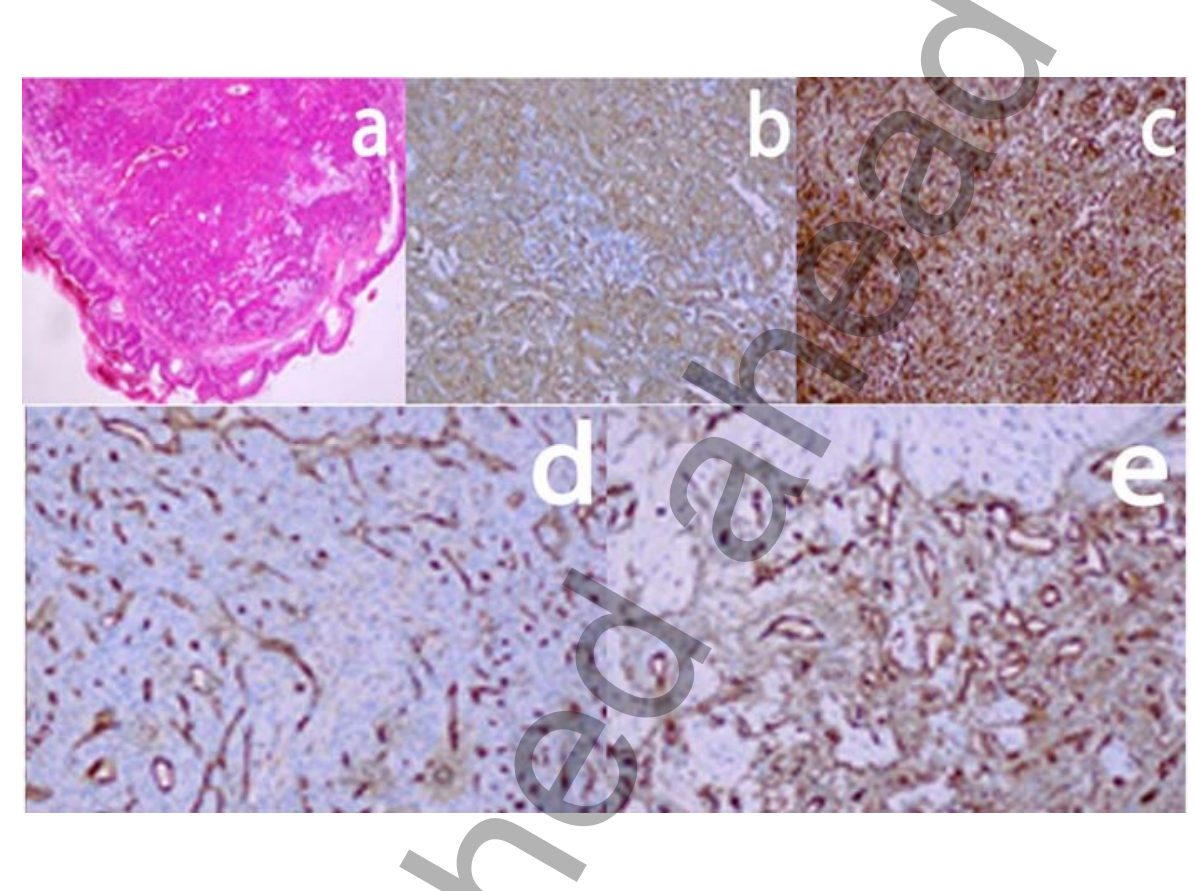

Figure 2-a: Hematoxylin-eosin staining reveals nodular lesion which is seperated from the squamous metaplastic epithelium of the respiratory tract with a clear neat zone. (original magnification $\times 10$ )

Figure 2-b: The results of immuno-histochemical staining SMA (+) in the lining cells of the lesion. (original magnification X 40)

Figure 2-c: The results of immuno-histochemical staining Vimentin $(+)$ in the cells that make up the lesion. (original magnification X40) 
Figure 2-d: The results of immuno-histochemical staining CD $31(+)$ in the vessel walls of the lesion. (original magnification X40)

Figure 2-e: The results of immuno-histochemical staining CD $34(+)$ in the vessel walls and peripheral cells of the lesion. (original magnification X 40)
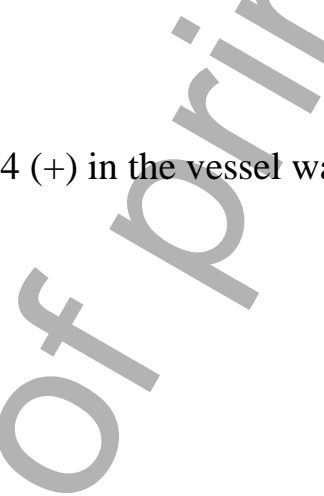

\section{and peripheral cells of the lesion. (original magnification $\mathrm{X} 40$ )}

